Ljubljana

\title{
SUR LES VALEURS DES TEMPS DU PASSE DANS LES LANGUES ROMANES
}

1. Le problème des valeurs des temps du passé dans les langues romanes est particulièrement complexe; qu'il soit attrayant, la quantité d'études , publiées ces dernières décennies, et les divergences d'opinions qui s'y manifestent en témoignent suffisamment. On ne saurait manquer d'être frappé par 1'opposition des vues concernant 1'existence supposée d'un système de l'aspect verbal, analogue à celui en vigueur dans les langues slaves.

L'intérêt est suscité par le fait mêmé de trouver dans les langues romanes, en comparaison à la situation dans les langues slaves, un nombre sensiblement supérieur de temps du passé; d'autre part, les langues romanes témoïgnent d'une richesse toute particulière dans ce domaine même par rapport au latin: toutes les formes périphrastiques, de la voix active, qu'elles aient remplacé les formes synthétiques latines ou bien qu'elles coexistent avec elles, sont des créations romanes.

Il semble, par conséquent, évident que le chercheur doit tenir compte de l'héritage dont les langues romanes sont redevable au latin: une étude purement synchronique risque de le priver de la connaissance de données historiques sans lesquelles la compréhension de la situation actuelle pourrait être obscure. Non que la constatation d'un fait du passé puisse à elle seule expliquer un fait présent: toutefois, la connaissance d'un état antérieur peut nous renseigner sur une situation critique qui a fini par provoquer un bouleversement du système. C'est ce qui s'est produit avec les temps du passé en latin. Heureusement, nous disposons d'une oeuvre fondamentale sur la naissance du prétérit périphrastique, celle de Thielmann. 1

Une analyse synchronique ne s'intéresse pas aux faits passés, elle cherche plutôt a découvrir le système actuel. Il est d'autant plus curieux de trouver tant d'études sur certains paradigmes verbaux: si, en sémantique; à partir de la mise en valeur des champs sémantiques il est presque obligatoire traiter les changements du sens d'un élément en vue du changement du système dont 1'élément en question fait partie, il est raisonnable d'attendre de meilleurs résultats des études qui traitent de tous les temps du passé, ainsi que du présent. Des monographies sur un paradigme verbal seulement sont nécessaires, voire indispensables, il faut admettre pourtant, qu'elles ne jettent qu'une lumière partielle.

1 Pl. Thielmann, Habere mit dem Part. Perf. Pass., ALLG 2, Leipzig 1885. 
2. Une étude diachronique part nécessairement de la constatation que les formes verbales périphrastiques, inconnues en latin littéraire, sont nées dans le latin parlé et que certains paradigmes coexistent avec les paradigmes hérités du latin et que d'autres s'étaient substitués aux formes synthétiques. Il n'est pas douteux que la première création d'une forme composée ait eu lieu avec le perfectum latin, temps du passé de beaucoup le plus important, hétérogène quant à son origine et polyvalent quant à sa valeur. Le perfectum latin condensait l'aoriste et le parfait grec. Il est possible, quoique non démontrable, que les formes verbales périphrastiques continuent les formes ou les structures existantes dans le latin populaire et dont une manifestation sporadique pourrait être relevée dans certains passages de Plaute, de Cicéron, de César. 2

Très séduisante est la métaphore selon laquelle le latin littéraire serait une espèce de glace qui aurait recouvert le fleuve de sorte qu' on ne l'apercevrait même pas; toutefois le fleuve, le latin parlé, langue de communication, coule sous la glace: son cours, c'est-à-dire, les innovations, les manifestations de la vie, peut apparaître comme une surprise. C'est une idée semblable qu'a eue, en regard de la création des formes périphrastiques, Herzog. Contre l'opinion formulée sur la base d'une documentation des plus rigoureuses par Thielmann, Herzog pense à une espèce de rivière intermittente: ${ }^{3}$ à cause d'un purisme qui ne tolère pas les formes périphrastiques, patrimoine de la langue parlée et, dans une faible mesure, de la langue écrite, elles n'apparaissent dans les textes latins que quelques siècles plus

2 Cfr. chez Plaute: Multa bona bene parta habemus, Trin. 347, ou chez Cicéron: in ea provincia pecunias magnas collocatas habent, De imp. Pomp. 18.

3 E. Herzog, Das -to-Partizip im Altromanischen, Beihefte zur ZRPh, XXVI, Wien 1910, p. 119: - Wir haben den Eindruck, dass ein Fluss von einem gegebenen Punkt an unterirdisch weiterfliesst, um endlich wieder, durch zugeflossene Bäche etwas verstärkt, an der Oberfläche zu erscheinen. Und so haben wir keinen anderen Ausweg, als die von Thielmann a limine abgewiesene Ansicht, dass das Zurücktreten von habere mit - toPartizip seit dem 1. Jahrh. n. Chr. ein 'scheinbares, bloss literarisches' war und dass die Konstruktion inzwischen ein immer wiederkehrender Teil des Vulgärlateins oder besser der lateinische Umgangssprache war. Und wenn wir fragen, warum die Schriftsprache dieser Konstruktion sichtbar ausweicht, so kann der Grund kein anderer sein, als der, dass damals das Verständnis für der ursprünglichen Sinn vollständig erlosch. Solange ein positum habeo seine eigene feine Nuance ausdrückte, war es literaturfähig, sowie Synonym von posui wurde, hatten die Puristen allen Grund es auszumerzen". 
tard, avec quelques exemples chez les Pères de l'Église et, d'une façon plus nette encore, dans la langue de Grégoire de Tours. 4

3. Les langues romanes ont hérité du latin à côté du perfectum simple un tour verbal qui servait, en latin parlé, à exprimer un état au présent: litteram scriptam habeo; un systeme de formes verbales périphrastiques, analogues à ce tour, $\mathrm{s}^{3}$ est créé, parallèle au système des formes simples. 5 A la valeur d'un état, valeur incontestable au moins pour le stade originaire du tour litteram scriptam habeo, s'est superposée la valeur de résultatif, l'état dans le présent entrainant 1'idée d'une action dans le passé ou, d'une façon plus général, dans le stade temporel antérieur à celui de l'état. Cette action ne peut pas être qu'accomplie dans le passé.

Si les langues romanes concordent pour avoir un système de paradigmes périphrastiques, parallèle à celui des temps simples, on constate un désaccord, quant à l' emploi du paradigme verbal, nommé dans la grammaire française le 'passé composé' et quant à son rapport avec le 'passé simple'. La forme simple a été, en français parlé, en maints dialectes italiens, en roumain, et ailleurs, supplantée par la forme périphrastique. Il s'agit du développement qu'Antoine Meillet dans son important article La disparition des formes simples du prétérit, de 1909, a condensé en deux points: 1) création de la forme composée du prétérit; 2) généralisation de cette forme aux dépens du prétérit simple. Je ne trouve pas que le terme "disparition" ne convienne pas. On conçoit sous ce terme la disparition syntaxique et sémantique. Que les formes du subjonctif du plus-que-parfait dans toutes les langues romanes et celles de 1'indicatif du même temps dans certaines langues romanes se soient conservées dans d'autres valeurs, c'est un fait, mais aucun des deux paradigmes n'a la valeur qu'il avait en latin. Autres paradigmes, comme le subjonctif du parfait, comme le futurum exactum, ont disparu morphologiquement aussi. Il n'en va pas de même du perfectum qui, jouissant d'une tout autre vitalité, continue son existence en tant qu'aoriste, action verbale pure. Le passé composé, conformément à son origine, sert à exprimer la notion d'un état au présent, un état qui est le résultat d'une action dans le passé.

4 Thielmann, op.cit., présente des oeuvres de Grégoire de Tours, Vitae Patrum et Historia Francorum des exemples convaincants: "Vescovum invitatum habes"; "Promissum habemus... nihil sine eius consilio agere".

5 Cfr. Si miles qui habebat jam factum testamentum, aliud fecisset, (Ulp. dig.). 
Si, aujourd'hui, dans les langues romanes la valeur d'un parfait n' est plus visible, sauf dans un très petit nombre de cas, 6 c'est que la forme composée s'est généralisée; dans certaines langues romanes, telles que le français, elle a relégué le passé simple à une variante écrite, voire littéraire du prétérit.

On reconnaîit donc difficilement la valeur originaire du passé composé; toutefois, c'est Lucien Foulet qui a donné dans sa Petite syntaxe de l'Ancien Francais des exemples qui permettent le mieux d'identifier dans la forme composée la valeur de parfait. L'auteur compare trois couples de propositions: "C'est un homme qui a beaucoup lu"; "Êtes-vous allé en Suisse?"; "Il a terminé son travail" avec "Il a beaucoup lu pendant sa maladie"; "Il est allé en Suisse aux grandes vacances dernières"; "Il a terminé son travail lundi" et conclut: - Si donc tout passé défini peut trouver un équivalent familier dans le passé indéfini correspondant, la réciproque n'est pas vraie. L'emploi du passé indéfini déborde celui du passé défini. C'est cet excédent qui constitue aujourd' hui le domaine du parfait.

Malgré la généralisation progressive du passé composé et bien qu'on ait des passages, à l'époque ancienne surtout, où les auteurs ne cherchaient qu'à varier la forme, nous disposons au cours de toute la littérature française d'exemples où nous pouvons être sûrs que l'auteur a voulu présenter un état dans le présent comme étant le résultat d'une action dans le passé.7 En voici quelques uns ot les deux formes se trouvent dans l'opposition aoriste: parfait: "Sept ans ad pleins qu'en Espaigne venimes: Jo vus conquis e Noples e Commibles; Pris ai Valterne e la tere de Pine"; "Ço sent RoIlanz, que s'espée li tolt, Uvrit les oilz, si li a dit un mot" (Chanson de Roland); "Tot est mudez, perdude a sa color" (Saint Alexis); "Quand on a tout perdu, que saurait-on plus craindre?" (Corneille). On trouve dans les grammaires françaises des exemples pris dans la langue d'aujourd'hui: "Quand il a déjeuné, il fait un tour dans le jardin" (Grévisse); "Chaque jour, quand j'ai déjeuné, je sors" (Damourette et Pichon).

6 Cfr. Dauzat, Grammaire raisonnée; Paris 1947, p. 214: L'idée d'achèvement est conservée avec quelques verbes: j'ai fini, j'ai vécu (sans complément).

J'ai essayé d'analyser les valeurs des deux formes du prétérit en italien dans Contributi alla storia del preterito nell' italiano, SAZU, Ljubljana 1970 .

7 La progression de la forme composée du prétérit n'est pas analogue dans toutes les langues romanes, le sort des deux formes verbales qui se disputent les valeurs du perfectum latin peut être bien différent. 
4. Ces derniers exemples nous intéressent plus particulièrement: il s'agit, il est vrai, d'une situation dans le présent, mais, en même temps, il s'agit d'une action à valeur extratemporelle. De cette valeur - et un des exemples donnés par Foulet, "Êtes-vous allé en Suisse?", serait de cette catégorie - une deuxième valeur du passé composé s'est développée, celle de l'action non ponctuelle, d'une action non concentrée en un point sur la ligne du temps, une action non condensée dans le temps: ${ }^{8}$ le passé composé, par cette valeur, se trouve dans une rigoureuse opposition avec le passé simple, lequel, aoriste, exprime une action close en elle-même et par conséquent temporellement bien déterminée.

Il est à noter que l'on retrouve souvent le passé composé indiquant une action non ponctuelle dans des propositions négatives. Un fait non réalisé est normalement présenté par la forme composée, sauf dans les cas où l'on nie expressement une seule action verbale. L'incompatibilité du passé simple avec une action niée fut sentie déja par Oudin, ${ }^{9}$ mais la condition première reste toujours celle de vouloir exprimer une action non ponctuelle, une action ligne, somme toute une action sans contour temporel précis. L'opposition aura bien entendu plus de relief là où l'on trouvera les deux formes dans des situations analogues, par ex.: "Il a conquis maintes belles isles de mer en cest archepel, où est ladicte Moree ... et aussi conquist presque toute 1'Albanye et Esclavonnye"; "Comme nostre Seigneur leur promit des ce qu'il feit 1'homme, et loyaulment 1' a tenu à toutes gens" (Commynes); "De tout temps les petits ont pati des sottises des grands (La Fontaine); "Le seul Childebert s'échappa et on ne sait pas ce qu'il est devenu: on dit qu'il pardonna à Mérovée, mais depuis on n'a plus entendu parler de lui" (Bossuet); "le docteur Bianchon a longtemps pratiqué la chirurgie avant de se livrer à la médecine" (Balzac); "cependant, parce que la nécessité du courage a toujours été inversement proportionnelle à la distance qui sépare les combattants" (Pagnol).

Si le caractère négatif de la proposition n'est pas une condition pour l'emploi du passé composé, il n'est pas nécessaire non plus que l'action se situe dans un passé proche: "Eschyle, le grand poète tragique athénien, a fait

8 Il n'est pas a espérer que la nomenclature des paradigmes verbaux puisse devenir uniforme, sauf si l'on se met d'accord pour employer des termes absolument arbitraires qui n'évoqueraient point le contenu, et porteraient exclusivement sur la forme. Pour cette opposition seulement, la vieille terminologie de la Grammaire du Port Royal (terminologie, employée aussi par Foulet) passé défini: passé indéfini semble excellente, mais on ne peut pas créer une terminologie basée sur une seule valeur de la forme quand elle en englobe plusieurs.

9 V. Ferdinand Brunot, Histoire de la langue française t. III, Paris 1909, pag. 583 , note: - . . on dit avec le simple Il y a trois sepmaines que je vous envoyay. Mais, si la phrase est négative, il faut le composé: il y a quinze jours que je ne vous ay veu. 
de longs séjours en Sicile: il fit représenter solennellement Les Perses à Syracuse en 470 et c'est à Géla qu'il mourut en 456", L'Histoire de la Sicile par Jean Huré, 'Que sais-je?', Paris 1957.10

5. Les deux formes du préterit peuvent, enfin, exprimer, respectivement, l'action éloignée et 1'action récente. Cette opposition est, chronologiquement, postérieure à la précédente. On trouve rarement, en ancien français, la forme composée pour exprimer une action appartenant à un passé récent; à l'époque moderne, en revanche, elle est en train de se substituer au passé simple. 11

Malgré quelques voix contraires, il n'y a pas de doute que le passé simple n'existe plus, et ceci depuis quelques siècles, dans le langage parlé de certaines langues romanes. On n'a pas encore trouvé d'explication valable, linguistique, pour justifier la perte du passé simple d'une part et son existence, de 1'autre, l'existence très tenace çà et là, dans la Peninsule ibérique, en Italie méridionale, surtout; du moins, peut-on bien justifier le lent progrès du passé composé: forme plus expressive, morphologiquement plus régulière, terminant par l'élément lexical. ${ }^{12} \mathrm{La}$ généralisation de la forme composée est un fait; il n'y a, évidemment, aucun lien logique entre la

10 Ajoutons un exemple pris dans la littérature italienne, cité déjà par Vockeradt, Lehrbuch der italienischen Sprache, Berlin 1878, par. 206. Pour l'emploi de la forme composée du préterit, à valeur d'action non ponctuelle dans le passé, le passage est à considérer comme paradigmatique: "Se il Padre degli uomini mi chiamasse a rendimento di conti, io gli dirò: non ho rapito il pane, non ho perseguitato l'infelice ... ho spartito il mio pane con l'indigente" (Foscolo, Le ultime lettere di Iacopo Ortis).

Rohlfs, Grammatica storica della lingua italiana e dei suoi dialetti, par. 673 , en citant ce passage, lui aussi, observe que le passé composé est employé avec cette valeur même dans les régions (Sicile, Calabre du Sud) qui, en dehors de cette valeur, ne connaissent que le passé simple.

11 "Pour moi, qui ai été élevé dans les départements du Cher et de 1'Allier", écrivait Antoine Meillet en 1909," .. la forme du passé défini $\mathrm{m}$ 'apparait comme barbare ou pédante, et je ne puis l'entendre dans la conversation ou la lire dans une lettre familière sans en être vivement choqué" dans 1'article Sur la disparition des formes simples du préterit, republié dans "Linguistique historique et linguistique générale", Paris 1948.

$12 \mathrm{~V}$. Charles Bally, Linguistique générale et linguistique française, Berne 1944, par. 5 et 340: "Le passé simple est le paradigme le plus touffu du mode indicatif". 
valeur d'un parfait et une action, ne serait-ce que la liaison entre un état au présent et une action dans le passé, entre le parfait j'ai écrit la lettre ('elle est maintenant écrite'), il est arrivé ('il est maintenant ici') et 1'aoriste j'ai écrit la lettre ce matin, il est arrivé il y a une demie heure. Cette généralisation bannit la forme simple du langage parlé et rend difficile, voire impossible d'identifier les diverses valeurs de la forme composée. Le passé simple, en français, reste du domaine de la langue littéraire. Nous nous devons ici d'examiner les opinions exprimées par Roland Barthes dans son oeuvre Le degré zéro de l'écriture. ${ }^{13}$ Le roman, pour Barthes, est un produit caractéristique de la société bourgeoise et le passé simple en est un élément essentiel. L' analyse de Barthes outrepasse décidément la réalité linguistique; dans un certain sens, elle évoque la vision idéaliste d'un Vossler, lequel liait le sort des deux formes du prétérit aux deux conceptions respectives de l'homme médiéval et de 1'homme moderne: une vision séduisante, et pourtant contredite par la réalité. Barthes pourrait objecter que les faits de langue ne sont pas toujours explicables par des raisons linguistiques; mais, une explication extralinguistique, nécessaire dans de nombreux cas, ne peut pas ne pas tenir compte du fait de langue. Barthes ne s'intéresse pas sur les raisons qui ont provoque la limitation de l'emploi ou la disparition du passé simple, et il est dans son droit: une analyse synchronique n'a pas pour but de rechercher les raisons qui ont conditionné un état linguistique. Toutefois, Barthes constate la décadence de la forme simple du prétérit comme un fait général, sans tenir compte du fait que dans d'autres langues romanes une telle assertion apparaît surprenante.

L'oeuvre littéraire qui, du fait de l'emploi du prétérit, a suscité le plus vif intéret semble L'Étranger. ${ }^{14}$ Camus recourt constanıment au passé com-

13 "Retiré du francais parlé, le passé simple, pierre d'angle du Récit, signale toujours un art; il fait partie d'un rituel des Belles-Lettres. Il n'est plus chargé d'exprimer un temps ... On s'explique alors ce que le passé simple du Roman a d'utile et d'intolérable: il est un mensonge manifesté ... La finalité commune du Roman et de 1'Histoire narrée, c'est d'aliéner les faits: le passé simple est l'acte même de possession de la société sur son passé et son possible ... Ceci doit être mis en rapport avec une certaine mythologie de l'universel, propre à la société bourgeoise, dont le Roman est un produit caractérisé ... le mécanisme du mythe, et le Roman - et dans le Roman, le passé simple - sont des objets mythologiques, qui superposent à leur intention immédiate, le recours second à une dogmatique", Le degré zéro de 1'écriture, Paris 1964, pag. 29, pag. 32.

14 V. Harald Weinrich, Tempus. Besprochene und erzählte Welt, Stuttgart 1964, pgs. 262-270. Albert Barrera-Vidal, La perspective temporelle dans "1'Étranger" de Camus et dans "la Familia de Pascual Duarte" de José Camilo Cela, ZRPh 84 (1968), pag. 309 ss. 
posé. Barrera Vidal a fait une analyse très suggestive, en mettant en comparaison les mots, le récit de deux condamnés à mort: celui dans l'oeuvre espagnole ne connaît, à quelques exceptions près, que des passés simples. $D$ 'après Barrera-Vidal ce condamné à mort parle avec un détachement de la vie, comme quelqu'un qui ne vit plus. On pourrait objecter que le passé simple est, en espagnol, la forme normalement employée dans un récit; qu' en italien, il en est de même et que les oeuvres d'Alberto Moravia (Conti romani, Il disprezzo, etc.), où l'auteur parle à la première personne, n'utilisent rigoureusement pour le récit que le passé simple. Il s'agit, à mon avis, d'un emploi neutre qui a sa justification dans la tradition, respectée aussi par la langue de la presse, et qui est rarement violée. ${ }^{15}$

En voulant justifier 1'emploi du passé composé dans L'Étranger, je penserais avant tout au passé composé comme un moyen stylistique: la forme composée, qui à l'origine exprime un état, exige après elle une pause, une suspension à peine perceptible, mais suffisante pour que le rythme rigide que crée la suite des actions liées dans une chaîne de passés simples soit brisée. ${ }^{16}$

II

6. J'ai parfois recouru dans l'exposé ci-dessus au terme d' "opposition", je n'ai pas employé le terme d' "opposition aspectuelle". Les deux formes verbales, dans des exemples cités, ne se trouvent pas dans 1'opposition aspectuelle, du moins, si l'on entend la notion de l'aspect verbal telle qu'elle est conçue dans des langues slaves. Dans un même verbe les deux formes du prétérit ne servent pas à exprimer action ou procès perfectíf ou imperfectif dans la même sphère temporelle; elles expriment, ou exprimaient, l'aoriste, action pure dans le passé, et le parfait, un résultatif, un

D'ailleurs, même pour les récits à la première personne, le passé simple est, dans la prose littéraire française, la forme habituelle. Ainsi dans les Souvenirs d'enfance de Marcel Pagnol.

15 Ainsi, dans Il padrone de Goffredo Parise. A noter, toutefois, que les événements se déroulent dans un centre industriel de l'Italie du Nord, dans un cadre, par conséquent, qui dans le langage parlé ne connaît absolument pas d'autre forme que le passé composé.

16 L'idée est déjà clairement énoncée par Bally, op.cit., pag. 353, note: Le passé composé ... présente les événements comme une série de faits accomplis; de là, bien souvent, le manque de vie qui caractérise la narration en langage courant. 
état dans le présent. Très instructif à cet égard est le terme qu'emploie pour 1'espagnol Andrés Bello: ante-presente. ${ }^{17}$

Les formes périphrastiques verbales, par leur formation même, ne peuvent être que des résultatifs dans des sphères du présent, du prétérit, du futur; le fait d'exprimer une action, par une conclusion logique, bien qu' inconsciente, est secondaire. L'état, par lui-même, est imperfectif; le fait qu'une forme nommée 'perfectum' serve à exprimer l'imperfectivité ne choque pas. Emile Benveniste, dans un article fondamental, 18 a posé comme point de départ de son analyse la constatation que les temps composés sont en même temps des parfaits par rapports aux temps simples, c'est-à-dire qu'ils présentent l'action comme accomplie, et qu'ils indiquent l'antériorité, toujours par rapport au temps simple correspondant. L'analyse de Benveniste est éminemment synchronique, la répartition des temps n'est pas essentiellement différente de celle de Meillet, bien que celle-ci soit limitée au latin et introduise une terminologie équivoque. 19 Mais Benveniste introduit une nouvelle distintion, qui oppose les notions de discours et de récit: les temps composés sont les temps du discours, les temps simples ceux du récit. Les exemples donnés, pourtant ne sont pas très clairs; pour Benveniste "quand il a fini son travail, il rentre chez lui est du discours, à cause du présent, et, aussi bien, de l'antérieur de présent; quand il eut fini ... il rentra est un énoncé historique, à cause de l'aoriste, et de I'antérieur d'aoriste". On comprend très bien ce que signifie 'récit', 'énoncé historique'; on comprend moins bien ce qu'il faut entendre par le terme de 'discours'. Dans l'exemple cité, le passé composé à valeur de parfait exprime un état dans le présent, c'est le présent de la principale qui en témoigne; ailleurs, la valeur du passé composé (par ex.: quand il a fini son travail, il est sorti) ne serait pas la même.

Weinrich a repris cette répartition, 20 avec des réserves et des modifications. Il classe les paradigmes verbaux en deux catégories bien distinctes, celle des formes verbales qui expriment une réflexion, une analyse, et celle qui comporte les formes servant à organiser le récit.

17 V. la réédition de sa Grammática de la lengua castellana, Buenos Aires 1960 , pag. 210 ss.: he cantado antepresente; hube cantado ante-pretérito; habré cantado ante-futuro; habria cantado ante-pos-pretérito.

18 Benveniste, Les relations de temps dans le verbe francais (1959) republié dans Problèmes de linguistique générale, Paris 1966.

19 Ainsi, dans son étude Sur les caractères du verbe (1920), republiée dans Linguistique historique et linguistique générale, Paris 1948.

20 Weinrich, op. cit., pag. 36 ss. 
Cette répartition, tres séduisante, restera toutefois partielle. Les temps en italien, par ex., sont présentés dans une novella de Pirandello, pag. 100: l'introduction, qui est une sorte d'analyse, recourt au passé composé, tandis que le récit proprement dit recourt au passé simple (on retrouve un procédé analogue dans le Décaméron). Dans l'introduction, dans le cadre de la nouvelle, on $n$ 'a pas affaire à un récit. Il s'agit d'une situation dans le présent: "Il Mèola ha rubato. Il Mèola s'è arricchito. Il Mèola probabillmente domani si metterà a far l'usurajo ..." Il s'agit, selon moi, d'un parfait, d'un perfectum praesens; cela ne veut pas dire que l'interprétation de Weinrich ne tienne pas ("Im Rahmen der Novelle wird jedoch der Betrug des Meola nicht erzählt, sondern besprochen".). Seulement, une telle interprétation n'est pas une interprétation de la valeur d'une forme verbale, elle fait partie d'une classification des emplois. L'auteur opère donc aussi avec des éléments extralinguistiques. Comme, du reste, lorsqu'il interprète l'emploi de l'imparfait dans le récit. 21

7. Benveniste soutient (op. cit., pag. 246), que les formes composées du prétérit ont surtout une valeur de parfait. En étudiant le sort du verbe en vieil italien Franca Ageno est parvenue aux mêmes conclusions. Son étude est d'autant plus précieuse que ses exemples présentent les formes verbales composées à valeur absolue, aussi dans des propositions désidératives ou impératives Se tu hai grazia divina, Fa che l'abbia conservata, (Bianco). Des exemples analogues ont été déjà aperçus de R. Ambrosini: La giovane cominciò la sua medicina e in brieve anzi il termine l'ebbe condotto a sanità (Boccaccio). 22

Il s'agit en effet de parfaits par rapport au présent ou au passé: c'est l'état qui est exprimé, à l'origine logique se trouve une action antérieure, 1'effet stylistique est puissant: le participe élimine l'hypothèse d'une éventuelle instantanéité dans le passé et ce n'est pas la durée qui est mise en évidence, mais la situation, l'état atteint.

21 Weinrich, op. cit., p. 159: - Das Imparfait ist in der Erzählung das Tempus des Hintergrunds, das Passé simple ist das Tempus des Vordergrunds.

22 Franca Brambilla Ageno, Questioni di aspetto en Il verbo nell' antico italiano, Milano-Napoli, 1964, pgs. 299-326. Riccardo Ambrosini, L'uso dei tempi storici nell'italiano antico (1955), republié dans 'Italia dialettale" 24, Pisa 1960-61.

Cfr., Stanko Škerlj, Osservazioni sul carattere, dotto o popolare che sia, dei costrutti "giunto che fu", "bello com'è" en "Atti dell'VIII Congresso internazionale di Studi Romanzi", II, Firenze 1969, pgs. 423-435 . 
8. Il est maintenant presqu'une nécessité de chercher à préciser, dans , toute la mesure du possible, la notion d'aspect verbal. Les travaux sur ce grand problème en général et y compris dans les langues romanes sont de plus en plus nombreux et les opinions absolument contradictoires. Le terme n'a pas d'importance; on sait qu'il est dû à un heureux contresens: 23 il s'agit d'une erreur de traduction du terme grec Eîo'og 'catégorie' dans les vieux traités médiévaux sur la langue slavonne, qui se servaient du terme vid, un emprunt adapté du grec. En traduisant la grammaire russe de Greč, le traducteur suisse Philippe Reiff, en 1828 , a traduit le terme vid par aspect.

"L'aspect est une catégorie inhérente au proces̀ envisagé sous l'angle de son déroulement": ${ }^{24}$ c'est une des définitions possibles, sans qu'elle puisse prétendre épuiser le sujet. Une chose semble pourtant acquise: la notion d'aspect doit être considérée comme distincte des modalités verbales, telles que la momentanéité, la durée, 1'itérativité, le début ou l'achèvement de I'action, etc. Elle ne s'appuie que sur l'opposition: perfectivité/imperfectivité.

Or, comme nous ne sommes pas directement intéressés ici par la situation dans les langues slaves, mais seulement par d'éventuelles correspondances avec les langues romanes, il suffira de dire ceci:

en principe, la majorité des verbes slaves sont d'aspect perfectif ou imperfectif; un très petit nombre ignore l'aspect;

dans un certain sens, à chaque verbe non-slave correspondent deux verbes slaves;

la majorité des verbes simples sont d'aspect imperfectif;

il existe des procédés de perfectivation et d'imperfectivation. D' une façon générale, la perfectivation d'un verbe imperfectif est obtenue par la préfixation (pisati/napisati 'écrire');

l'imperfectivation d'un verbe perfectif est obtenue seulement par l'infixation (kupiti/kupovati 'acheter'). De plus, ce sont surtout les alternances vocaliques et consonantiques, du radical ou du suffixe qui peuvent déterminer l'aspect (izbirati/izbrati 'choisir').

23 Mazon, L'aspect verbal dans les langues slaves, IV Congrès International des Slavistes, Moscou 1958.

24 Claude Vincenot, Essai de grammaire slovène, Ljubljana 1975, pag. 221. 
9. Il existe un bon nombre de travaux qui se sont proposés de rechercher la catégorie d'aspect dans des langues romanes. Les options prises par les chercheurs vont d'une totale dénégation 25 jusqu'à une partielle reconnaissance de l'existence de l'aspect ou au moins de modalités de verbe. Il convient peut-être ici de citer Eugenio Coseriu26 qui fait siennes les paroles de E. Buyssens (1958): "Toutes les langues connaissent un système hybride de temps et d'aspect; le degré d'hybridation varie, mais nulle part on ne trouve un système aspectuel pur ou un système temporel pur".

Il n'est pas à mettre en doute le fait que les langues romanes possèdent des possibilités d'exprimer, jusqu'à un certain degré, l'opposition aspectuelle perfectivité: imperfectivité, en général, avec d'autres moyens que les langues slaves. Les langues romanes n'ont pas hérité du latin des débris des oppositions aspectuelles comme iacēre - iácere; cubāre - (suc)cúmbere. Toutefois, l'opposition aspectuelle peut être rendue:

a) par deux verbes, sémantiquement opposés, qui, par la nature même, sont ou perfectifs ou imperfectifs: chercher/trouver;

b) par des moyens qui tiennent au verbe même, et plus précisement

๙) par le jeu des temps du verbe, notamment par l'imparfait en opposition avec d'autres temps du passé. C'est le cas pour 1'imparfait dans sa valeur originaire, celle d'imperfectivité, ce qui ne s'accorde pas nécessaire-

25 Cfr. Emidio de Felice, Problemi di aspetto nei più antichi testi francesi, Vox Romanica, 16, Bern 1957, pag. 51: - Ne risulta ... che 1'as petto flessionale, nell' antico francese, non costituisce un valore grammaticale unitario e costante, e comunque mai un sistema coerente di opposizioni.

L. Jenaro Maclennan, El problema del aspecto verbal, Madrid 1962, pag. 31: - . . la introducción de términos como perfectivo/imperfectivo para aludir a conceptos semánticos más que a hechos morfológicos reales, términos sólo aplicables a las lenguas eslavas, de cuyo seno jamás debieron salir.

Weinrich, op. cit., pag. 152: - Ein unglücklicher Begriff: "Aspekt". D'ailleurs, déjà Meillet, Sur les caractères du verbe (1920): - La catégorie de l'aspect est plus concrète que celle du temps, et, au cours de l'histoire des langues indo-européennes, on voit 1'aspect perdre de l'importance, le temps en gagner ... Les langues romanes $n$ ' ont pas gardé ce système, elles ont laissé tomber tout ce qui avait valeur d'aspect, et elles n' ont gardé que la valeur temporelle. (En "Linguistique historique et linguistique générale", pag. 185).

26 E. Coseriu, Das romanische Verbalsystem, Tübingen 1976. 
ment avec la notion de durativité. C'est pourquoi un énoncé comme il sonnait une heure ne surprend pas: 27 le verbe à l'imparfait n'exprime pas la durée, il exprime l'imperfectivité. Avec certains verbes, les valeurs aspectuelles sont particulièrement visibles (savoir, avoir, connaître): contre l'imparfait, le passé simple et le passé composé servent à exprimer une action perfective.

Il est à noter que l'imparfait connaît d'autres emplois encore, exprime l'itérativité, action non réalisée ('imperfectum de conatu' latin). A ncter aussi qu' un verbe slave d'aspect imperfectif au passé n'est pas toujours traduisible par l'imparfait roman: une détermination temporelle quelconque empêche, dans les langues romanes, l'emploi de l'imparfait. Le passé simple en Le spectacle dura deux heures; Henri IV regna vingt ans aurait comme correspondance le prétérit d'un verbe imperfectif dans les langues slaves.

Le plus-que-parfait d'après le système, esquissé par Benveniste, sert à exprimer un "parfait de l'imparfait" ou un état dans le passé, et, dans le même temps, l'action pure peut être exprimée par le plus-que-parfait. Il y a bien, surtout en italien, la possibilité de rendre la notion d'une action, sans aucune nuance d'antériorité.

B) Des changements dans le verbe même sont possibles aussi par la préfixation, mais seulement avec un nombre restreint de suffixes et encore le même suffixe n'est pas toujours un facteur de la valeur aspectuelle. Le sens peut changer: courir/acourir; les deux verbes forment une opposition aspectuelle (en slovène avec teči/priteči nous trouvons une formation analogue), le verbe préfixé exprimant 1'achèvement d'une action imperfective du simplex. Dans le couple battre/abattre le sens tellement différent des deux verbes nous empêche $d^{\prime} y$ voir une opposition aspectuelle laquelle, certainement, est à l'origine d'une telle composition. On constate, par contre, une opposition aspectuelle dans les couples verbe intransitif: verbe réflexif (avec préfixe), comme dormir/s'endormir; aller/s'en aller. L'italien, plus riche que le français quant à la préfixation, compte un certain nombre de verbes dérivés de 1'adjectif: impallidire, arrossire. La modalité incohative est commune à tous ces dérivés.

c) les valeurs aspectuelles peuvent être exprimées aussi par des périphrases verbales. Aux couples je savais: j'ai su; j'avais: j'ai eu, j'ai recu dans le passé on peut opposer au présent je sais: je viens à savoir, vengo a sapere.

27 Weinrich, op. cit., pag. 153. 
C'est surtout le tour je suis en train de parler (plus fort encore, peut etre, le tour italien sto parlando et les tours analogues dans 1'ibéroroman) qui rend de façon claire l'aspect imperfectif du verbe. De plus, l'actualisation de l'action verbale est accentuée. Dans les langues romanes, ce tour n'est pas encore un élément de morphologie verbale. 28 Toutefois, la polarisation du présent simple et du présent composé est en train de devenir un fait dans certaines situations; l'emploi du syntagme ne sera donc plus un fait de style. Le syntagme est d'autant plus destiné à se grammaticaliser au présent, car il s'oppose au présent, forme plurivalente; dans le passé, certes, le syntagme j'étais en train de ...; stavo parlando n' est qu' un stylème par rapport à l'imparfait. 29

Il est à noter que la forme verbale périphrastique je suis en train de ... et ses équivalents dans d'autres langues romanes n'est pas possible avec les verbes lesquels, par nature, expriment un état, comme être, avoir, durer, comme stare; ni avec des verbes impersonnels comme il semble, il paraît. Ce sont les verbes qui dans les langues slaves n' expriment pas de notion d'aspect. Les verbes perfectifs admettent la périphrase avec être en train de, stare etc. Certes, ils acquièrent une valeur imperfective, due à la présence du verbe (sémi) auxiliaire être, stare, en même temps, le syntagme devient porteur des modalités de l'action: futur immédiat, itérativité, etc. C'est pourquoi certains verbes, décidément momentanés, comme trouver, ne connaissent pas un tel syntagme.

28 Cfr. Jerzy Kurylowicz, L'apophonie en indo-européen, Wroclaw 1956, pag. 27: Le tour périphrastique je suis en train d'écrire ne fait pas encore partie de la conjugaison française orthodoxe. L'expression "présent simple" a été deja utilisé; v. R. Lenz, La oración y sus partes, par. 301; A. Sauvageot, Français écrit - français parlé, Paris 1962, pag. 93.

29 Devoto, Introduzione alla grammatica, Firenze 1941, pag. 153, voit dans les formes simples 'valore durativo normale' et dans les formes périphrastiques 'valore durativo intenso'. La forme périphrastique actualise 1 'action en la rendant contemporaine à l'acte de la parole; cette certitude manque dans l'emploi du présent, dit "simple". Sur ce point, il n'y a pas de parallélisme avec le couple dans l'imparfait: j'étais en train de parler, stavo párlando sont des tours plus expressifs que les imparfaits "simples"; cela n'enlève toutefois pas la valeur d'imperfectivité aux formes je parlais, parlavo. - Il a exprimé une opinion différente, en voyant dans l'imparfait une forme stylistiquement non marquée, l'italianiste norvégien Kolbjorn Blücher. V. son oeuvre: Studio sulle forme ho cantato, cantai, cantavo, stavo cantando, Bergen 1970. 
D'autres périphrases verbales seront employées pour exprimer la modalité incohative; c'est le cas des verbes commencer, se mettre à. C'est l'expressivité de telles périphrases (s'asseoir - être assis; mettersi a sedere - star seduti) qui rend préférable, et parfois indispensable leur emploi.

d) A l'aspect dans les langues slaves peut aussi correspondre une construction non-verbale. L'opposition singulier/pluriel (indéterminé) peut être exploitée pour rendre la notion d'opposition aspectuelle perfectif/imperfectif: au couple en slovène migniti/migati correspond faire signe/faire des signes. 30

10. Étant donné que la catégorie de 1'aspect existe dans les langues slaves, il est tout naturel de tenter d'en déceler également l'existence dans les langues romanes. Les conclusions des chercheurs sont contradictoires et vont d'une totale dénégation jusqu'à une (peut être trop) audacieuse utilisation de la terminologie, empruntée à la philologie slave. Il n'est pas possible, toutefois, de nier l'existence de certaines manifestations aspectuelles dans les langues romanes. Si elles ne constituent pas un système solide comme en le trouve dans le slave, on peut dire en revanche que le système verbal roman apparaît beaucoup plus riche. Il est trop rigide de dire que ces manifestations ne représentent qu'un choix stylistique; au moins, l'emploi de l'imparfait contre celui du passé simple ou du passé composé, l'emploi des verbes sémantiquement opposés, l'emploi des verbes préfixés et des périphrases verbales, opposeés aux formes simples, entrent à coup sûr dans les catégories d'aspect. Il n'est pas pensable qu'un système aspectuel . complet puisse naître dans les langues romanes; toutefois, grâce aux exigences du style, des stylèmes pourraient devenir un patrimoine de la grammaire, passer donc de l' individuel au collectif, de la parole à la langue, avec une vague saveur de début de systémisation.

30 Vincenot, op. cit., pag. 226. 


\section{Povzetek}

\section{O VREDNOSTIH GLAGOLSKIH OBLIK ZA PRETEKLOST V ROMANSKIH JEZIKIH}

Želja, da bi ugotovili resnične vrednosti glagolskih oblik za preteklost $\mathrm{v}$ romanskih jezikih, tako priča število objavljenih del, je iz desetletja $v$ desetletje večja. Zlasti je vidna pri tistih raziskovalcih, ki imajo v svojem jeziku drugačen sistem, tako na primer pri romanistih slovanskega rodu, ali pri tistih, ki se s slovanskimi jeziki posebej ukvarjajo. Zanimanje je upravičeno, saj skušajo sistem glagolskega vida, naj jim je že vrojen ali pa sicer dobro poznan, zaslediti $\mathrm{v}$ sistemu glagolskih časov. Ena od bitnih razlik med romanskim in slovanskim glagolom je ravno ta, da se bogastvo paradigem prvega izravnava $z$ aspektnimi izraznimi možnostmi slovanskega glagola.

Avtor vidi $\mathrm{v}$ primerjavi romanskih in slovanskih jezikov pravilno metodo, zlasti, ker sta sistem glagolskih časov in sistem aspektnih vrednosti prepletena; meni, da je pravilna sodba belgijskega jezikoslovea Buyssensa (Revue Belge de Philologie et d'Histoire 36, Bruxelles 1958), ki pravi, da ni jezika, ki bi poznal samo aspektni sistem, pa tudi ne takega, ki bi poznal samo sistem glagolskih časov; različna pa je stopnja prepletanja. Upati je, da bo kontrastivna analiza prinesla novih dognanj.

Ni mogoče misliti, da bi $\mathrm{v}$ romanskih jezikih osnovne aspektne opozicije perfektivnost/imperfektivnost sploh ne zaznavali. Ta opozicija, in razen nje glagolske modalnosti ("vrste glagolskih dejanj". po Toporišiču, Slovenska slovnica, str. 288), $v$ romanskih jezikih biva. Ni izrazljiva z morfološkimi sredstvi, čeprav je že latinščina nekaj tega imela, pač pa jo zaznavamo drugače.

Predvsem imamo tudi v romanskih jezikih glagole, ki so občuteni kot perfektivni ali kot imperfektivni, npr. iskati/najti. Posredno kažejo na naravo glagola tisti primeri, kjer pri prevajanju ne najdemo ustreznega glagola $v$ romanskih jezikih, recimo za slov. glagol poiskati, in se je treba začeti $\mathrm{k}$ opisu, ali pa uporabiti dva glagola, od katerih bo eden izražal proces in drugi dovršnost, oziroma končno modalnost.

Najpomembnejše izrazno sredstvo pa se vendar skriva $v$ glagolu: od preteklih časov je imperfekt $\mathrm{v}$ ostri aspektni opoziciji zlasti $\mathrm{s}$ tistima dvema, ki ju francoska slovnica imenuje passé simple in passé composé.

Seveda ni vsak imperfekt že kar izraz nedovršnosti; ta glagolska paradigma lahko izraža posamična dejanja, pa tudi posamična dejanja, vezana $v$ verigo dogodkov. Nadalje se pretekli čas slovanskih nedovršnih glagolov ne 
dá zmeraj prevesti $z$ imperfektom: dejanja in procese $v$ stavkih kot Predstava je trajala dve uri; Henrik IV je vladal dvajset let vidijo romanski jeziki kot časovno določena, to pa izključuje uporabo imperfekta.

Kot $\mathrm{v}$ slovanskih jezikih je mogoče s prefiksi iz nedovršnih glagolov tvoriti dovršne, vendar tak prefiks nima zmeraj te naloge. Večkrat se pri tvorbi zabriše osnovni pomen, prim. fr. battre: abattre 'posekati'. V vseh romanskih jezikih je mogoče iz enostavnih nedovršnih glagolov tvoriti dovršne s pomočjo prefiksa, glagol pa samo po obliki postane povraten, prim. it. dormire: addormentarsi 'zaspati'; šp. ir: irse 'oditi'.

Romanski jeziki imajo na voljo vrsto glagolskih perifraz, ki izražajo eno od aspektnih vrednosti; najpomembnejše so uporabe $z$ nedoločnikom, in še bolj z gerundijem, npr. šp. estoy hablando ali estou falando $\mathrm{v}$ brazilski portugalščini; estou a falar v evropski portugalščini. Ta perifraza izraža trajajoče dejanje ali stanje, kar seveda izraža tudi imperfekt, a perifraza dejanje aktualizira. Uporaba perifraze je torej stilistično sredstvo, vendar je prav mogoče, da se bodo take perifraze iz stilne rabe počasi vrinile $v$ morfosintaktični sistem. Če trdnega sistema aspektnih opozicij $\mathrm{v}$ romanskih jezikih ni, pa ni mogoče dvomiti, da se opozicije, do neke mere občutene, vendarle pojavljajo. 